Published in final edited form as:

J Am Chem Soc. 2017 October 25; 139(42): 14897-14900. doi:10.1021/jacs.7b09306.

\title{
Directed $\gamma-\mathrm{C}\left(s p^{3}\right)-\mathrm{H}$ Alkylation of Carboxylic Acid Derivatives through Visible Light Photoredox Catalysis
}

\author{
Dian-Feng Chen ${ }^{\dagger}$, John C. K. Chu ${ }^{\ddagger}$, and Tomislav Rovis ${ }^{\dagger,}{ }^{*}$ \\ tDepartment of Chemistry, Columbia University, New York, New York 10027, United States \\ ‡Department of Chemistry, Colorado State University, Fort Collins, Colorado 80523, United States
}

\begin{abstract}
Visible light photoredox catalysis enables direct $\gamma-\mathrm{C}_{s p} 3 \mathrm{H}$ alkylation of saturated aliphatic carbonyl compounds. Electron-deficient alkenes are used as the coupling partners in this reaction. Distinguished site selectivity is controlled by the predominant 1,5-hydrogen atom transfer (HAT) of an amidyl radical generated in situ.
\end{abstract}

\section{Graphical Abstract}

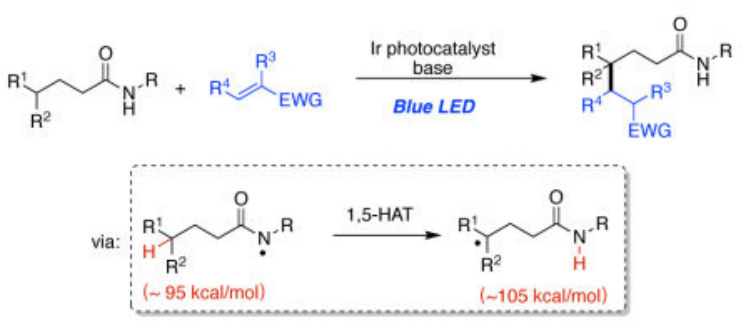

\begin{abstract}
Strategies that selectively functionalize unactivated $\mathrm{C}_{s p} 3 \mathrm{H}$ bonds in the presence of multiple other sites are of intense current interest. ${ }^{1}$ Directed reactions are common in this milieu, often via transition metal catalysis. ${ }^{2}$ Among diverse functionality capable of directing $\mathrm{C}-\mathrm{H}$ activation, carbonyls are attractive due to their ubiquity and versatility. $\beta$-Functionalization of aliphatic carbonyl compounds has been facilitated by robust organo- ${ }^{3}$ or transition-metal catalysis. ${ }^{4}$ Although some progress has been made, $\gamma$-C-H functionalization remains a formidable challenge. A literature survey disclosed two strategies for addressing this issue. First, Corey, ${ }^{5}$ Chatani, ${ }^{6} \mathrm{Chen}^{7}$ and $\mathrm{Yu}^{8}$ pioneered primary $\gamma$-C-H arylation, alkynylation, olefination and intramolecular amination reactions based on sterically controlled formations of six-membered metallacycles (Fig 1a). Methylene and methine groups are not amenable to these reactions. Second, intramolecular hydrogen atom abstraction by an amidyl radical, ${ }^{9}$ in a 1,5 -fashion (i.e. modified Hofmann-Löffler-Freytag reaction ${ }^{10}$ ), generates a $\gamma$-carbon
\end{abstract}

\footnotetext{
*Corresponding Author: tr2504@ columbia.edu.

Notes

The authors declare no competing financial interest.

Supporting Information.

The Supporting Information is available free of charge on the ACS Publications website.
} 
centered radical. These radicals largely end up as halogenated ${ }^{11}$ or cyclized product, ${ }^{12}$ due to high reactivity of the excess halogen reagents within the systems.

Very recently, we $\mathrm{e}^{13}$ and Knowles ${ }^{14}$ reported selective $\mathrm{C}_{s p} 3^{-\mathrm{H}}$ bond abstraction and alkylation with electron-deficient alkenes relying on photoredox catalysis ${ }^{15}$ with selectivity governed by a 1,5 -hydrogen atom transfer $(\mathrm{HAT})^{16}$ reaction (Fig $1 \mathrm{~b}$ ). The reacting center was thus delivered from a pendant amine functionality which was oxidized by the excited state of the photocatalyst. The net transformation may be viewed as remote functionalization of an aliphatic amine. With this success, we turned our attention to remote functionalization of other aliphatic systems using complementary functional groups to deliver selectivity.

We anticipated that the formation of an amide bond on a carboxylic acid would afford an opportunity to create a different $\mathrm{N}$-centered radical after oxidation, but it still might allow for selective 1,5-HAT to activate remote sites. The immediate challenge was that a trifluoroacetamide, which we used in our amine-directed functionalization, could not be used for carboxyl-directed functionalization. At the outset of this work, it was not clear what functionality would be required since it would have to fulfill several criteria in the successful generation/translocation of nitrogen radicals. It is well documented ${ }^{16}$ that the high electrophilic character of the amidyl radical ${ }^{9}$ is crucial for hydrogen atom abstraction. Therefore, an appropriate protecting group (usually electron-deficient) on nitrogen is required for three reasons: 1) to acidify the $\mathrm{N}-\mathrm{H}$ proton to enable mild deprotonation, 2) to give the resulting amidyl anion reasonable oxidation potential for single electron transfer (SET), and 3) to provide a higher $\mathrm{N}-\mathrm{H}$ bond dissociation energy $(\mathrm{BDE}){ }^{17}$ relative to that of $s p^{3} \mathrm{C}-\mathrm{H}$ bonds, ultimately allowing for efficient abstraction.

An initial survey of common N-protecting groups on 4-methylpentanamide revealed ethoxycarbonyl as being optimal (see SI for details). Methyl methacrylate (MMA) was used

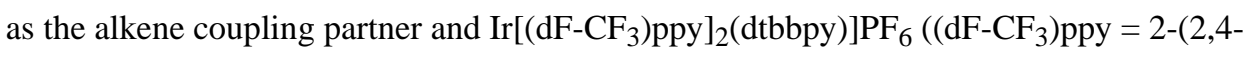
difluorophenyl)-5-(trifluoromethyl)pyridine; dtbbpy $=4,4^{\prime}$-di-tert-butyl-2,2' -bipyridine) ${ }^{18}$ was chosen as the photocatalyst. We were delighted to find that the reaction of $1 \mathbf{a}$ in the presence of saturated aq. $\mathrm{K}_{3} \mathrm{PO}_{4}$ and blue light provided the desired product 3aa in $76 \%$ NMR yield (Table 1, entry 1). Superior yield (74\% isolated, entry 5) was obtained by using 1.1 equiv of $\mathrm{K}_{3} \mathrm{PO}_{4}$ and 1:1 mixed DMF/t-AmylOH. Control experiments suggested that the photocatalyst, base and light were all essential for a successful $\gamma-\mathrm{C}-\mathrm{H}$ alkylation reaction of carbamate 1a (entry 6).

With optimized conditions in hand, we next investigated the alkene scope with amide 1a or $\mathbf{1 b}$ as the substrate (Scheme 1). Reactions of 1a with acrylates bearing a-substituents, including alkyl and aryl groups, proceed well, delivering products $\mathbf{3 a b}-\mathbf{3 a d}$ in good yields (68-72\%). Acrylates without a-substituents couple with 1a providing products 3ae and 3af in slightly lower yields, likely a consequence of competitive aza-Michael addition as well as oligomerization (see SI for details). Allyl acrylate engages in reactivity as the desired product 3ag is exclusively obtained in $62 \%$ isolated yield. Methacrylonitrile is also a suitable partner for this reaction (3ah, 62\% yield). This protocol was then successfully applied to other electron-deficient alkenes, such as methyl vinyl ketone, acrylamide, vinyl sulfone and vinyl phosphonate, when amide $\mathbf{1 b}$ is used. Products $\mathbf{3 b i} \mathbf{- 3} \mathbf{b l}$ were isolated in 
moderate to good yields. $\beta$-substituents on more electron-deficient alkenes (e.g. dimethyl maleate) result in an incomplete reaction, as $\mathbf{3 b m}$ was obtained in only $47 \%$ yield.

The amide scope was examined next (Scheme 2). Substrates with tertiary $\gamma-\mathrm{C}-\mathrm{H}$ bonds (BDE $\sim 95 \mathrm{kcal} / \mathrm{mol}$ ) are well tolerated, giving alkylated products $3 \mathbf{c f}-\mathbf{3 g e}$ in good yields (Scheme 2a). Tunable mono- or di-alkylation of substrates bearing activated secondary $\mathrm{C}-\mathrm{H}$ bonds (BDE $\sim 90 \mathrm{kcal} / \mathrm{mol}$ ) is also possible (3ie - 3je' $\mathbf{e}^{\prime}$ ). This could be achieved by simply altering the ratio between the amide and alkene starting materials. A pharmacologically relevant $\gamma$-aminobutyric acid derivative gives mono-alkylated product $3 \mathbf{k l}$ in moderate yield (38\%). Unactivated secondary C-H bonds remain a challenge (3hi, 14\% yield). Next, amide substrates possessing two or more potential hydrogen abstraction sites were examined (Scheme 2c). Gratifyingly, 1,5-HAT process was found to outcompete other pathways. ${ }^{19}$ As a result, products $\mathbf{3 l f}-\mathbf{3 r f}$ are formed in moderate to good yields. Functional groups such as chloride (3ee), BocN-H (3ne) and acetal (3se) are also tolerated. A steroidal derivative with multiple stereogenic centers and tertiary $\mathrm{C}-\mathrm{H}$ bonds exclusively furnishes product 3ue in moderate yield. It is noteworthy that a glucose-derived radical intermediate undergoes alkylation with modest diastere oselectivity (3tl).

We further interrogated the selectivity of 1,5-HAT when presented with two distinct tertiary $\mathrm{C}-\mathrm{H}$ bonds (Eq 1-3). In all cases investigated, regioselectivity is high. With substrates $\mathbf{1 v}$ and $1 \mathrm{w}, \mathrm{HAT}$ occurs on the $\mathrm{C}-\mathrm{H}$ bond distal to the $\sigma$-withdrawing oxygen functionality, presumably for electronic reasons. Substrate 1x, on the other hand, has two electronically similar tertiary C-H bonds, Eq 3. HAT is still completely selective, delivering product 3xe in $54 \%$ yield. The two competitive HAT reactions presumably proceed via bicyclic transition states ([4.4.0] and [3.3.1]), with the former being preferred for reasons of ring strain. ${ }^{20}$
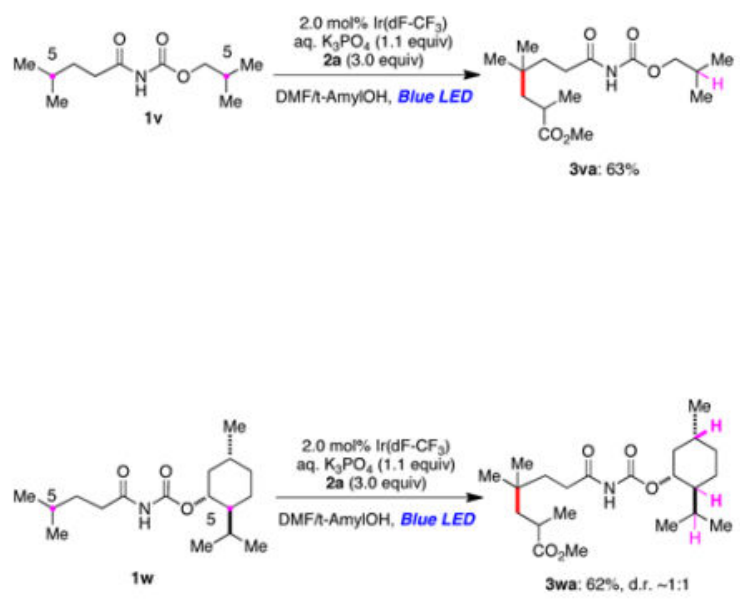


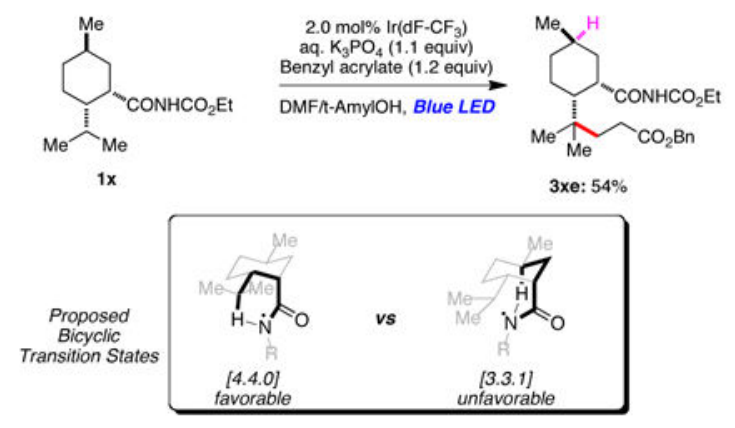

A possible mechanistic pathway is shown in Figure 2. Deprotonation of $1 \mathbf{a}(\mathrm{p} K a \sim 11.0$ in $\mathrm{H}_{2} \mathrm{O}, \sim 16.5$ in DMSO $)^{21}$ with $\mathrm{K}_{3} \mathrm{PO}_{4}\left(p K a \sim 12.4 \text { in } \mathrm{H}_{2} \mathrm{O}\right)^{22}$ provides potassium amide $\mathbf{I}$. A Stern-Volmer experiment (see SI for details) reveals the feasibility of single electron oxidation of $\mathbf{I}\left(E_{1 / 2}{ }^{\text {red }}=+1.04 \mathrm{~V}\right.$ vs saturated calomel electrode (SCE) in DMF) by the excited state of $\left.\operatorname{Ir}\left[\left(\mathrm{dF}_{-} \mathrm{CF}_{3}\right) \text { ppy }\right]_{2}(\mathrm{dtbbpy})\right] \mathrm{PF}_{6}\left(E_{1 / 2}{ }^{\text {red }}\left[* \mathrm{Ir}^{\mathrm{III}} / \mathrm{Ir}^{\mathrm{II}}\right]=+1.21 \mathrm{~V}\right.$ vs SCE in $\mathrm{MeCN}),{ }^{18}$ which furnishes amidyl radical II. A subsequent energetically favorable 1,5-HAT $\left(\mathrm{N}-\mathrm{H}\right.$ BDE $\sim 105.5 \mathrm{kcal} / \mathrm{mol}^{23}$ vs tertiary $\gamma$-C-H BDE $\sim 95 \mathrm{kcal} / \mathrm{mol}$ ) gives nucleophilic $\gamma$ carbon radical III, which is then trapped by electron-deficient alkenes (e.g. methyl methacrylate), leading to the formation of a new carbon radical IV. Single electron reduction of IV $\left(E_{1 / 2}{ }^{\text {red }}=-0.73 \sim-0.59 \mathrm{~V}\right.$ vs SCE in $\left.\mathrm{MeCN}\right){ }^{24}$ by the $\mathrm{Ir}^{\mathrm{II}}$ species $\left(E_{1 / 2}{ }^{\mathrm{red}}\left[\mathrm{Ir}^{\mathrm{III}} / \mathrm{Ir}^{\mathrm{II}}\right]=\right.$ -1.37 vs SCE in $\mathrm{MeCN}),{ }^{18}$ followed by protonation, affords product 3aa, thereby also regenerating the ground-state $\mathrm{Ir}^{\mathrm{III}}$ catalyst. $^{25}$

In summary, we have developed a visible light photoredox-catalyzed $\gamma$-alkylation of saturated aliphatic carboxylic acid derivatives with a variety of electron-deficient alkenes. Efficient in situ generation of the amidyl radical, and subsequent translocation in a 1,5fashion, ensures excellent site-selectivity. We have further delineated substrate scope and shown that synthetically useful levels of regioselectivity may be attained for more complex substrates.

\section{Supplementary Material}

Refer to Web version on PubMed Central for supplementary material.

\section{Acknowledgments}

We thank NIGMS (GM125206) for support. JCKC acknowledges a Croucher Scholarship from the Croucher Foundation, Hong Kong. We thank Matthew Shores (CSU) for the use of his actinometer.

\section{References}

1. White MC. Science. 2012; 335:807. [PubMed: 22344434]

2. He J, Wasa M, Chan KSL, Shao Q, Yu J-Q. Chem Rev. 2017; 117:8754. [PubMed: 28697604]

3. Fu Z, Xu J, Zhu T, Leong WWY, Chi YR. Nat Chem. 2013; 5:835. [PubMed: 24056339] 
4. For excellent examples, see: Giri R, Maugel N, Li JJ, Wang DH, Breazzano SP, Saunders LB, Yu JQ. J Am Chem Soc. 2007; 129:3510. [PubMed: 17335217] Wasa M, Engle KM, Yu JQ. J Am Chem Soc. 2009; 131:9886. [PubMed: 19580277] He J, Li S, Deng Y, Fu F, Laforteza BN, Spangler JE, Homs A, Yu JQ. Science. 2014; 343:1216. [PubMed: 24626923] Zhang FL, Hong K, Li TJ, Park H, Yu JQ. Science. 2016; 351:252. [PubMed: 26816374] Chen G, Gong W, Zhuang Z, Andrä MS, Chen YQ, Hong X, Yang YF, Liu T, Houk KN, Yu JQ. Science. 2016; 353:1023. [PubMed: 27701111] Wu QF, Shen PX, He J, Wang XB, Zhang F, Shao Q, Zhu RY, Mapelli C, Qiao JX, Poss MA, Yu JQ. Science. 2017; 355:499. [PubMed: 28154075] Pirnot MT, Rankic DA, Martin DBC, MacMillan DWC. Science. 2013; 339:1593. [PubMed: 23539600]

5. Reddy BVS, Reddy LR, Corey EJ. Org Lett. 2006; 8:3391. [PubMed: 16836413]

6. Ano Y, Tobisu M, Chatani NJ. Am Chem Soc. 2011; 133:12984.

7. (a) He G, Zhang SY, Nack WA, Li Q, Chen G. Angew Chem, Int Ed. 2013; 52:11124.(b) He G, Zhang SY, Nack WA, Pearson R, Rabb-Lynch J, Chen G. Org Lett. 2014; 16:6488. [PubMed: 25487778]

8. (a) Li S, Chen G, Feng CG, Gong W, Yu JQ. J Am Chem Soc. 2014; 136:5267. [PubMed: 24666182] (b) Li S, Zhu RY, Xiao KJ, Yu JQ. Angew Chem, Int Ed. 2016; 55:4317.

9. (a) Zard SZ. Chem Soc Rev. 2008; 37:1603. [PubMed: 18648685] (b) Sutcliffe R, Griller D, Lessard J, Ingold KU. J Am Chem Soc. 1981; 103:624.

10. For a review, see: Wolff ME. Chem Rev. 1963; 63:55.

11. Liu T, Myers MC, Yu JQ. Angew Chem, Int Ed. 2017; 56:306.

12. (a) Liu T, Mei TS, Yu JQ. J Am Chem Soc. 2015; 137:5871. [PubMed: 25915558] (b) Richers J, Heilmann M, Drees M, Tiefenbacher K. Org Lett. 2016; 18:6472. [PubMed: 27978701] (c) Wappes EA, Fosu SC, Chopko TC, Nagib DA. Angew Chem, Int Ed. 2016; 55:9974.

13. Chu JCK, Rovis T. Nature. 2016; 539:272. [PubMed: 27732580]

14. Choi GJ, Zhu Q, Miller DC, Gu CJ, Knowles RR. Nature. 2016; 539:268. [PubMed: 27732585]

15. For excellent reviews, see: Prier CK, Rankic DA, Mac-Millan DWC. Chem Rev. 2013; 113:5322. [PubMed: 23509883] Skubi KL, Blum TR, Yoon TP. Chem Rev. 2016; 116:10035. [PubMed: 27109441]

16. Čeković Ž. J Serb Chem Soc. 2005; 70:287.

17. Relative homolytic bond dissociation energies ( $\triangle \mathrm{BDEs}$ ) compared to acetamide ( $\mathrm{p} K_{a}=25.5$ in DMSO, $E_{O X}\left(\mathrm{~N}^{-}\right)=+0.73 \mathrm{~V}$ vs $\mathrm{Ag} / \mathrm{AgCl}$ in $\left.\mathrm{MeCN}\right): \Delta \mathrm{BDEs}(\mathrm{N}-\mathrm{H})=1.37 \Delta \mathrm{p} K_{a}(\mathrm{~N}-\mathrm{H})+23.06$ $\Delta E_{O X}\left(\mathrm{~N}^{-}\right)$. Bausch MJ, David B, Prasad V, Wang L-H, Vaughn A. J Phys Org Chem. 1992; 5:1.

18. Lowry MS, Goldsmith JI, Slinker JD, Rohl R, Pascal RA Jr, Malliaras GG, Bernard S. Chem Mater. 2005; 17:5712.

19. Other pathways, see: Nechab M, Mondal S, Bertrand MP. Chem Eur J. 2014; 20:16034. [PubMed: 25345694] Chen K, Richter JM, Baran PS. J Am Chem Soc. 2008; 130:7247. [PubMed: 18481847]

20. Compare the strain energies of a [4.4.0] bicyclic system (cis-decalin - $4.1 \mathrm{kcal} / \mathrm{mol}$ ) with that of a [3.3.1] system ( $9.6 \mathrm{kcal} / \mathrm{mol})$; see: Greenberg A, Liebman JF. Strained Organic Molecules: Organic Chemistry: A Series of Monographs. 38Academic PressNew York1978; :72.

21. (a) Arnett EM, Harrelson JA Jr. J Am Chem Soc. 1987; 109:809.(b) Bordwell FG, Harrelson JA Jr, Lynch TY. J Org Chem. 1990; 55:3337.(c) Bordwell FG, Fried H. J Org Chem. 1991; 56:4218.

22. Chiong, HA. e-EROS Encyclopedia of Reagents for Organic Synthesis. 2010. Potassium Phosphate.

23. $E_{\mathrm{OX}}(\mathrm{I})=1.18 \mathrm{~V}$ vs $\mathrm{Ag} / \mathrm{AgCl}$ in DMF (see SI for details).

24. Bortolamei N, Isse AA, Gennaro A. Electrochim Acta. 2010; 55:8312.

25. A low quantum yield $(6.3 \%)$ and an on/off study suggest that the chain propagation mechanism is unlikely to be dominant in this reaction; see SI. 
(a) Corey/Chen/Yu:

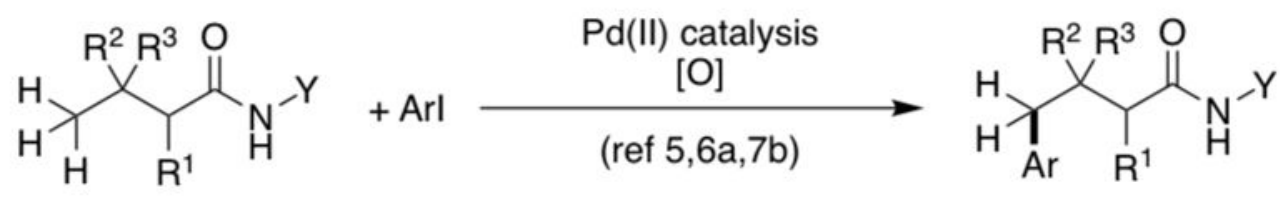

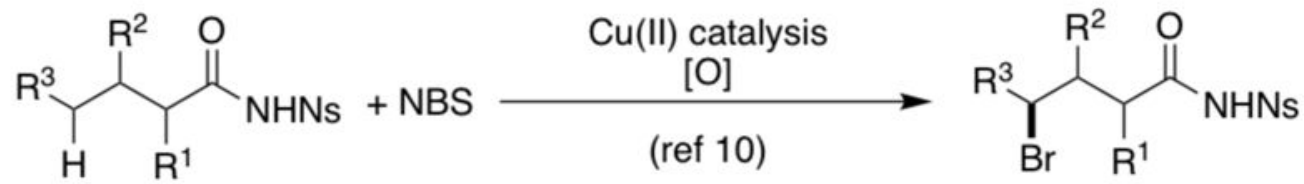

(b) Rovis/Knowles:

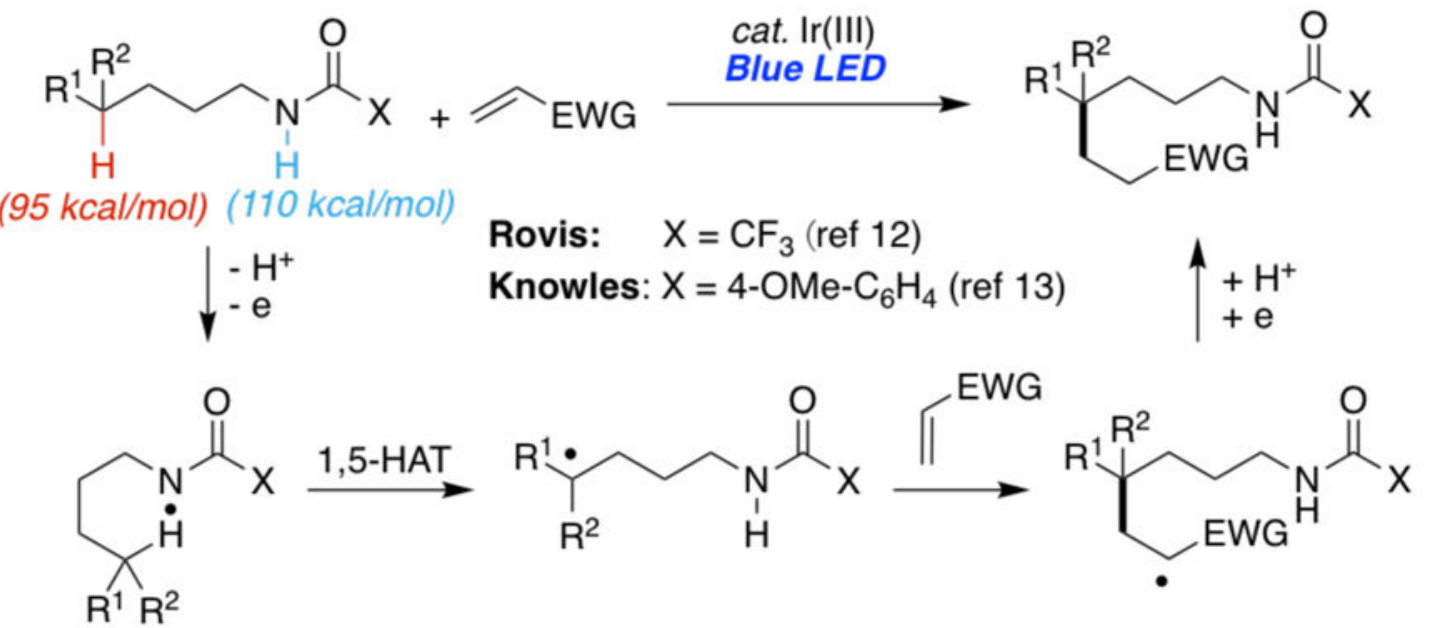

This Work:

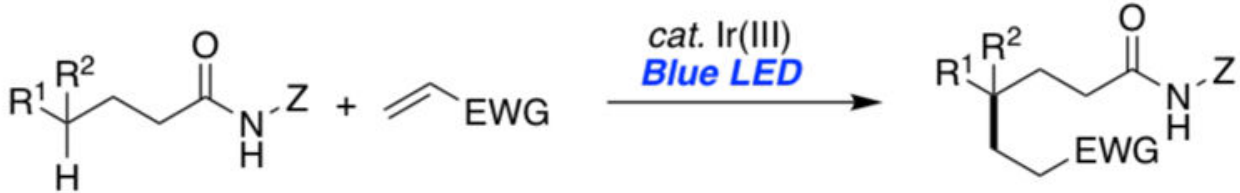

Figure 1.

Remote $\mathrm{C}_{\mathrm{sp} 3}-\mathrm{H}$ Functionalization 


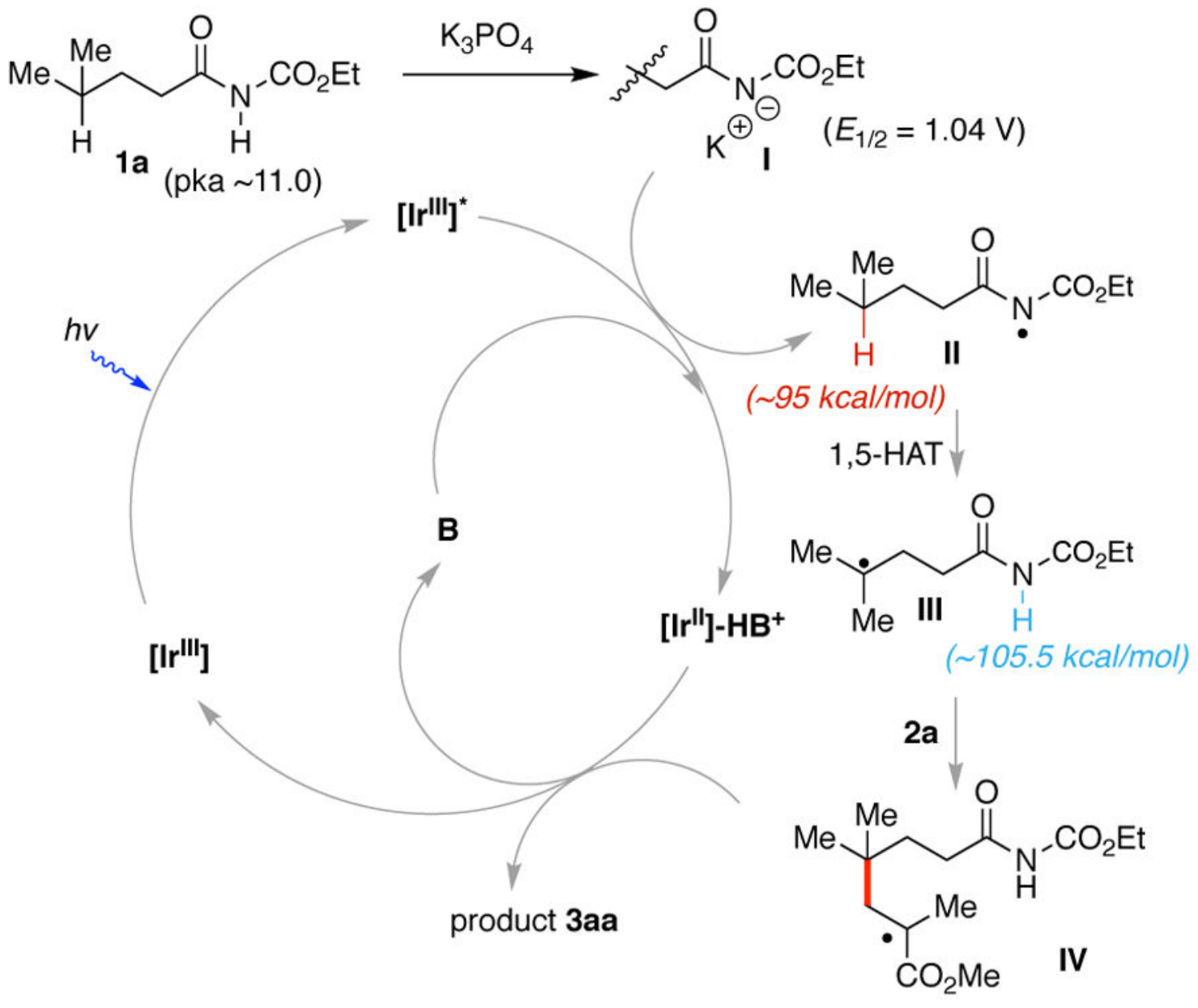

Figure 2.

Proposed Mechanism. 


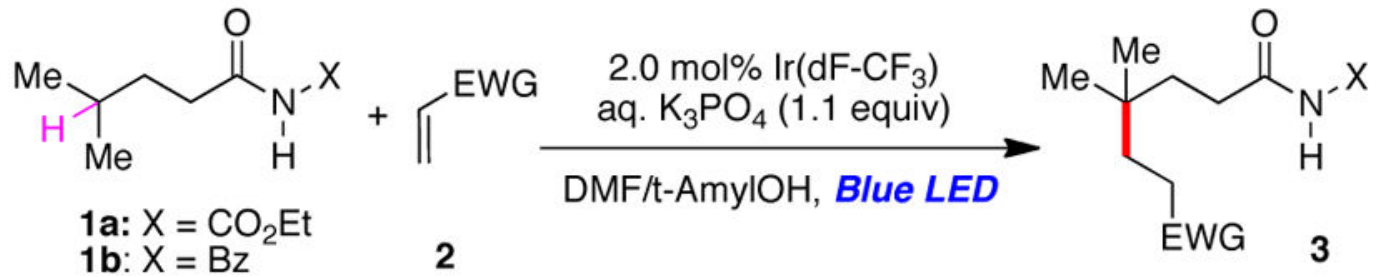<smiles>[R]OC(=O)CCC(C)(C)CCC(=O)NCCOCC(=O)NC(=O)OCC</smiles><smiles>C=CCOC(=O)CCC(C)(C)CCC(=O)NC(=O)OCC</smiles>

3ab: $R^{1}=B n, R^{2}=M e, 68 \%$;

3ac: $R^{1}=M e, R^{2}=B n, 70 \%$;

3ae: $\mathrm{R}^{3}=\mathrm{Bn}, 61 \%$;

3ad: $\mathrm{R} 1=\mathrm{Ph}, \mathrm{R}^{2}=\mathrm{Me}, 72 \%$;

3af: $R^{3}={ }^{t} \mathrm{Bu}, 66 \%$;

3ag: $62 \%$<smiles>CCOC(=O)NC(=O)CCC(C)(C)CC(C)C#N</smiles>

3ah: $62 \%$<smiles>CC(C)(C)CCNC(=O)CCC(C)(C)C</smiles>

3bk: $55 \%$<smiles>CC(=O)NC(=O)CCC(C)(C)CCC(C)C</smiles>

3bi: $44 \%$<smiles>CCCCNC(=O)CCC(C)(C)CCOCC</smiles>

3bl: $78 \%$<smiles>CC(=O)NCCCC(C)(C)CCC(=O)OCc1ccccc1</smiles>

3bj: $60 \%$

Scheme 1.

Alkene Scope ${ }^{a, b}$

${ }^{a} \operatorname{Ir}\left(\mathrm{dF}-\mathrm{CF}_{3}\right)(2.0 \mathrm{~mol} \%), \mathrm{K}_{3} \mathrm{PO}_{4}$ (1.1 equiv), 1 (1.0 equiv), 2 (1.2-3.0 equiv), 1:1 mixed DMF/t-AmylOH, $34 \mathrm{~W}$ blue LED, $40{ }^{\circ} \mathrm{C}, 12 \mathrm{~h}$. ${ }^{b}$ Isolated yields. 

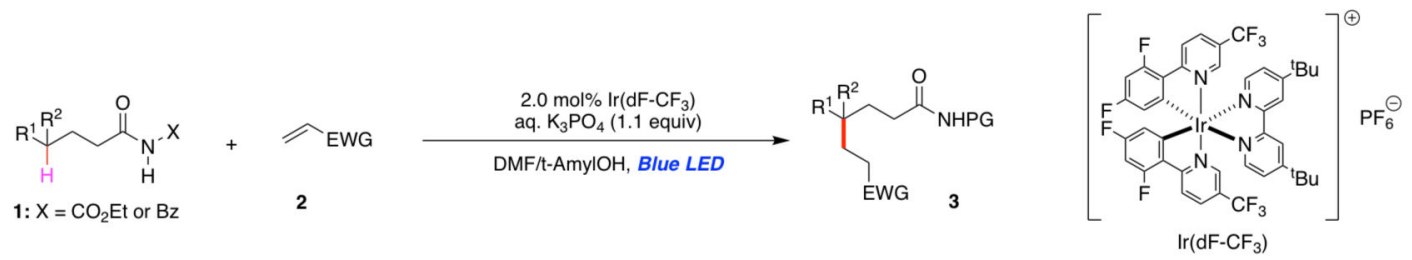

a) Tertiary C-H Bonds

$\overbrace{\mathrm{CO}_{2}{ }^{\prime} \mathrm{Bu}}^{\mathrm{O}} \overbrace{\mathrm{NHCO}_{2} \mathrm{Et}}^{\mathrm{Et}}$

3cf: $68 \%$<smiles>CCOC(=O)NCCCC1(CC(C)C(C)=O)CCCC1</smiles>

3da: $67 \%$

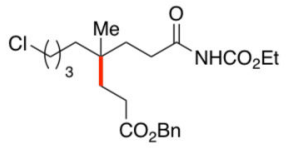

3ee: $54 \%$

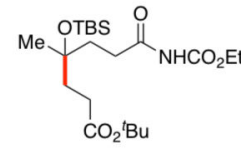

3ff: $71 \%$<smiles>CCOC(=O)NC(=O)CCC1(CCC(=O)Oc2ccccc2)CCCN1</smiles>

3ge: $51 \%$

\section{b) Secondary C-H Bonds}

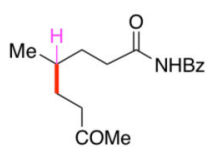

3hi: $14 \%$

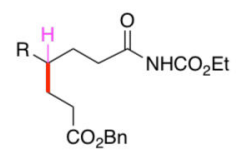

$3 \mathbf{i e}^{d}: \mathrm{R}=\mathrm{OMe}, 77 \%$ $3 \mathrm{je}^{d}: \mathbf{R}=$ OTBS, $49 \%$

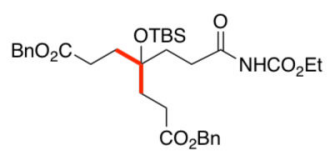

$3 \mathrm{je}^{\mathrm{e}}: 36 \%$

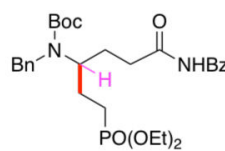

3kl: $38 \%$ (76\% brsm) c) Site-Seletivity

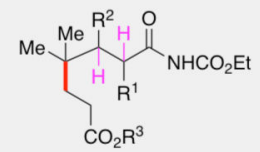

3lf: $R^{1}=M e, R^{2}=H, R^{3}={ }^{t} B u, 66 \%$; 3mf: $R^{1}=H, R^{2}=M e, R^{3}=' B u, 57 \%$; 3ne: $R^{1}=H, R^{2}=$ NHBoc, $R^{3}=B n, 43 \%$
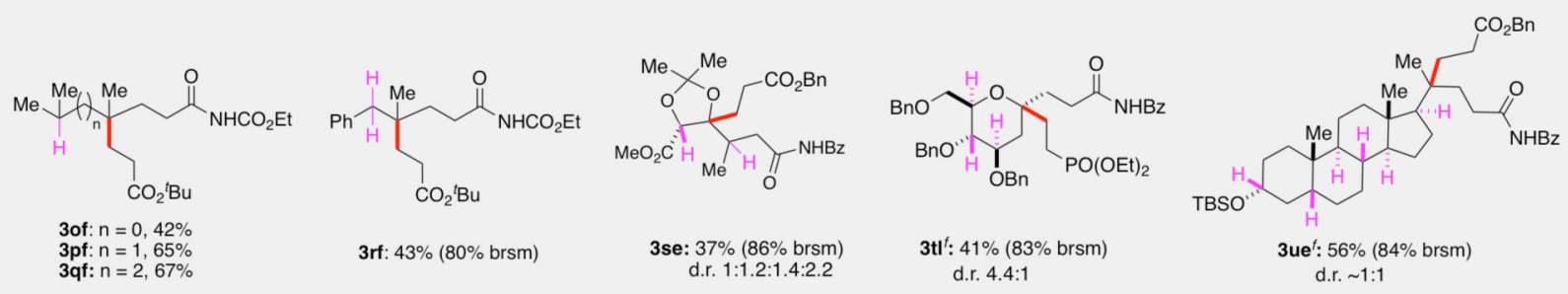

3ue $: 56 \%$ (84\% brsm) d.r. $\sim 1: 1$

\section{Scheme 2.}

Amide Scope ${ }^{a, b, c}$

${ }^{a} \operatorname{Ir}\left(\mathrm{dF}-\mathrm{CF}_{3}\right)(2.0 \mathrm{~mol} \%), \mathrm{K}_{3} \mathrm{PO}_{4}$ (1.1 equiv), 1 (1.0 equiv), 2 (1.2-3.0 equiv), 1:1 mixed DMF/t-AmylOH $(1.0 \mathrm{~mL}), 34 \mathrm{~W}$ blue LED, $\mathrm{N}_{2}, \sim 40^{\circ} \mathrm{C}, 12 \mathrm{~h}$. ${ }^{b}$ Isolated yields. ${ }^{c}$ d.r. was determined by the crude ${ }^{1} \mathrm{H}$ NMR. ${ }^{d} 1.2$ equiv of amide and 1.0 equiv of benzyl acrylate were used. ${ }^{e} .0$ equiv of benzyl acrylate was used. ${ }_{4} .0 \mathrm{mmol} \%$ of $\operatorname{Ir}\left(\mathrm{dF}-\mathrm{CF}_{3}\right)$ was used. $\operatorname{Ir}\left(\mathrm{dF}_{-} \mathrm{CF}_{3}\right)=\operatorname{Ir}\left[\left(\mathrm{dF}-\mathrm{CF}_{3}\right) \text { ppy }\right]_{2}(\mathrm{dtbbpy}) \mathrm{PF}_{6}$. 


\section{Table 1}

Optimization $^{a}$

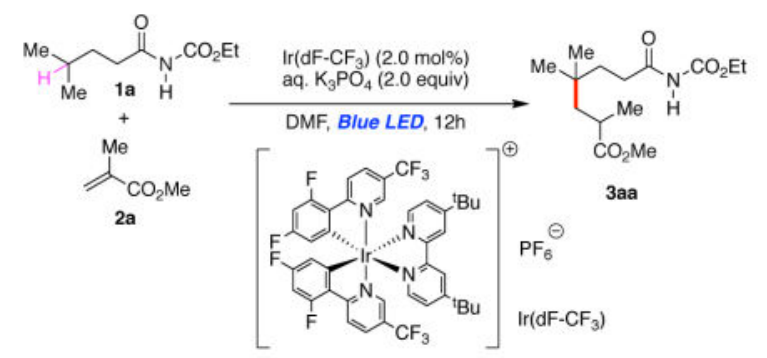

\begin{tabular}{cccc}
\hline Entry & Base & Solvent & Yield (\%) ${ }^{\boldsymbol{b}}$ \\
\hline 1 & $\mathrm{~K}_{3} \mathrm{PO}_{4}$ & $\mathrm{DMF}$ & 76 \\
2 & $\mathrm{Cs}_{2} \mathrm{CO}_{3}$ & $\mathrm{DMF}$ & 66 \\
3 & Quinuclidine & $\mathrm{DMF}$ & 0 \\
4 & $\mathrm{~K}_{3} \mathrm{PO}_{4}{ }^{c}$ & $\mathrm{DMF}$ & 83 \\
$5^{d}$ & $\mathrm{~K}_{3} \mathrm{PO}_{4}{ }^{c}$ & $\mathrm{DMF} / \mathrm{t}-\mathrm{AmylOH}(1: 1)$ & $84\left(74^{\mathcal{e}}\right)$ \\
$6^{d, f}$ & $\mathrm{~K}_{3} \mathrm{PO}_{4}{ }^{c}$ & $\mathrm{DMF} / \mathrm{t}-\mathrm{AmylOH}(1: 1)$ & 0 \\
\hline
\end{tabular}

${ }_{\mathrm{Ir}(\mathrm{dF}-\mathrm{CF} 3)}(2.0 \mathrm{~mol} \%)$, aq. $\mathrm{K}_{3} \mathrm{PO}_{4}$ (2.0 equiv), 1a (1.0 equiv), 2a (5.0 equiv), DMF, $34 \mathrm{~W}$ blue LED, $40^{\circ} \mathrm{C}, 12 \mathrm{~h}$.

$b_{\text {Yield determined by }}{ }^{1} \mathrm{H}$ NMR using trimethoxybenzene as the internal standard.

$c_{1.1}$ equiv.

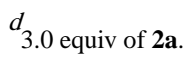

Isolated yield.

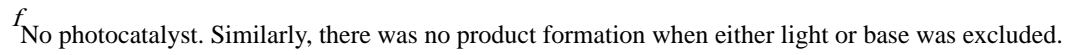

